\title{
Avaliação da Rede Cegonha: devolutiva dos resultados para as maternidades no Brasil
}

\author{
Evaluation of the Rede Cegonha: feedback of results for Brazilian \\ maternity hospitals
}

Luiza Beatriz Ribeiro Acioli de Araújo Silva (https://orcid.org/0000-0001-7095-0659) ${ }^{1}$

Antonia Angulo-Tuesta (https://orcid.org/0000-0002-3231-5918) ${ }^{2}$

Maria Teresa Rossetti Massari (https://orcid.org/0000-0002-4841-4208) ${ }^{1}$

Liliane Cristina Rodrigues Augusto (https://orcid.org/0000-0002-8164-5577) ${ }^{3}$

Laura Lamas Martins Gonçalves (https://orcid.org/0000-0003-2213-095X) ${ }^{4}$

Carla Kristiane Rocha Teixeira da Silva (https://orcid.org/0000-0002-4167-9342) ${ }^{5}$

Natali Pimentel Minoia (https://orcid.org/0000-0002-3505-0572) ${ }^{6}$

${ }^{1}$ Instituto Nacional de Saúde da Mulher, da Criança e do Adolescente Fernandes Figueira, Fiocruz. Av. Rui Barbosa 716, Flamengo. 22250-020 Rio de Janeiro RJ Brasil. luacioli23@gmail.com

${ }^{2}$ Programa de PósGraduação em Ciências e Tecnologias em Saúde, Faculdade de Ceilândia. Brasília DF Brasil. ${ }^{3}$ Organização PanAmericana de Saúde. Brasília DF Brasil.

${ }^{4}$ Centro de Ciências da Saúde, Universidade Federal do Maranhão. São Luís MA Brasil.

${ }^{5}$ Secretaria Estadual de

Saúde do Rio de Janeiro. Rio de Janeiro RJ Brasil.

${ }^{6}$ Ministério da Saúde.

Brasília DF Brasil.

\begin{abstract}
This paper describes and analyzes the process of providing feedback on the results of the second evaluation cycle of good practices of delivery and birth care in maternity hospitals linked to the Rede Cegonha, a Ministry of Health strategy implemented in 2011 to improve obstetric and neonatal healthcare and management. This is a qualitative study based on the documentary analysis of 27 reports from the states and the Federal District referring to the feedback workshops with 1.641 participants, $40 \%$ of whom were professionals and managers of the maternity hospitals evaluated, 25\% of state representatives, $20 \%$ of municipal health secretariats and 15\% of federal representatives. Around $46 \%$ of maternity hospitals' action plans in 11 states were received from January to August 2019. The results show the challenge of incorporating the monitoring and evaluation processes in these maternity hospitals' daily lives due to structural issues in institutional culture. This situation interferes with the local systematic analysis of information and the implementation of national evaluation cycles with the swift and continuous feedback of the results since access to secondary national data is non-existent in good delivery care practices.
\end{abstract}

Key words Health assessment, Health management, Healthcare models, Rede Cegonha, Delivery
Resumo Este artigo descreve e analisa o processo de devolutiva dos resultados do $2^{\circ}$ ciclo avaliativo das boas práticas na atenção ao parto e nascimento em maternidades vinculadas à Rede Cegonha, estratégia do Ministério da Saúde implementada em 2011, com enfoque no potencial de contribuição para a melhoria da gestão e atenção obstétrica e neonatal. Trata-se de estudo qualitativo de análise documental de 27 relatórios dos estados e do Distrito Federal referentes às Oficinas de Devolutiva com 1.641 participantes, sendo $40 \%$ de profissionais e gestores das maternidades avaliadas, 25\% de representantes estaduais, 20\% das secretarias municipais de saúde e $15 \%$ de representantes da esfera federal. Entre janeiro e agosto de 2019, foram recebidos $46 \%$ dos planos de ação de maternidades de 11 estados. Os resultados demonstram o desafio de incorporar os processos de monitoramento e avaliação no cotidiano destas maternidades, por questões estruturais da cultura institucional. Essa situação interfere na análise local de informações de forma sistemática e na realização de ciclos nacionais de avaliação com retorno dos resultados de forma ágil e contínua, uma vez que o acesso a dados nacionais secundários é inexistente no caso das boas práticas de assistência ao parto e nascimento.

Palavras-chave Avaliação em saúde, Gestão em saúde, Modelos de assistência à saúde, Rede Cegonha, Parto 


\section{Introdução}

O número de mortes maternas de um país constitui excelente indicador de sua realidade social e econômica, pois reflete a qualidade da assistência médica, as iniquidades de gênero e étnico-raciais, as desigualdades regionais, a determinação política e o fortalecimento do sistema de saúde pública ${ }^{1}$. A realidade enfrentada pelos gestores do SUS (Sistema Único de Saúde) em relação à morte materna no Brasil é complexa. Há expressivas diferenças regionais quanto aos seus determinantes e às estratégias para sua redução. Fazem parte desta realidade os desafios de superação das barreiras institucionais, sociais e culturais na atenção ao parto e nascimento baseados em evidências científicas e a garantia de direitos das mulheres ${ }^{2-5}$.

Quando se analisa a população de mulheres que apresenta complicações relacionadas à gestação, observa-se que significativa parcela dessa população sofre demoras na assistência, seja em relação à deteç̧ão precoce das complicações, ao uso de intervenções apropriadas ou ao processo de coordenação entre os níveis de atenção do sistema de saúde $e^{4,6,7}$. Do ponto de vista tecnológico e político-institucional, existem intervenções preventivas ou terapêuticas efetivas para a redução da mortalidade materna disponíveis no Brasil e realizadas em alguns territórios locais. Desde 1984, ações, programas e estratégias foram implementados no país, com alguns avanços ${ }^{8,9}$.

$\mathrm{Na}$ década de 1990, a razão de mortalidade materna era de 143/100.000 nascidos vivos (nv), chegando a 72/100.000 nv nos anos 2000. No período de 2000 a 2010 essa razão oscilou entre 72 e 69/100.000 nv, ocorrendo nova oscilação entre 2010 e 2017 entre 62 e 65 óbitos/100.000 nv ${ }^{10}$ (Gráfico 1).

Vale ressaltar que, mesmo com a redução de mais de 50\% dos óbitos maternos, o Brasil ainda está longe de alcançar os 30 óbitos para cada 100.000 nv, meta pactuada nos Objetivos do Desenvolvimento Sustentável ${ }^{11}$. Desde então, diversas políticas e marcos regulatórios nacionais foram implementados, dentre eles, o Pacto Nacional para Redução da Mortalidade Materna e Neonatal, o Pacto pela redução da mortalidade infantil no Nordeste e Amazônia Legal, a Política Nacional de Atenção Integral à Saúde da Mulher e a Política Nacional de Atenção Integral à Saúde da Criança, que apontam para princípios e diretrizes em consonância com os padrões internacionais ${ }^{12}$. $\mathrm{O}$ desafio diz respeito à mobilização de gestores e trabalhadores de saúde e, também, à capacidade de gerar sustentabilidade às condi- ções das práticas de saúde para se efetivar as recomendações nacionais e internacionais.

Diante deste cenário, o Ministério da Saúde (MS), em parceria com o CONASS (Conselho Nacional de Secretários de Saúde) e CONASEMS (Conselho Nacional de Secretarias Municipais de Saúde) instituiu a Rede Cegonha (RC), através da Portaria GM/MS 1.459 de 2011 (revogada pela Portaria de consolidação no 3 e no 6 de 2017) ${ }^{13,14}$. Esta normatização baseou-se nas diretrizes de Redes de Atenção à Saúde (RAS) no SUS ${ }^{15}$ e de atenção ao parto e nascimento da Organização Mundial de Saúde ${ }^{16}$. Estas últimas mais tarde atualizadas nos Protocolos Clínicos e Diretrizes Terapêuticas Nacionais para Cirurgias Cesarianas ${ }^{17}$ e Parto Normal ${ }^{18}$ no SUS.

Na regulamentação da RC, foram estabelecidos três objetivos: (i) fomentar a implementação de novo modelo de atenção à saúde da mulher e à saúde da criança com foco na atenção ao parto, ao nascimento, ao crescimento e ao desenvolvimento da criança de zero aos vinte e quatro meses; (ii) organizar a Rede de Atenção à Saúde Materna e Infantil para garantir acesso, acolhimento e resolutividade; e (iii) reduzir a mortalidade materna e infantil com ênfase no componente neonatal. As suas Diretrizes dizem respeito à: (i) garantia do acolhimento com avaliação e classificação de risco e vulnerabilidade, (ii) ampliação do acesso e melhoria da qualidade do pré-natal; (iii) garantia de vinculação da gestante à unidade de referência e ao transporte seguro; (iv) garantia das boas práticas e segurança na atenção ao parto e nascimento; (v) garantia da atenção à saúde das crianças de zero a vinte e quatro meses com qualidade e resolutividade; e (vi) garantia de acesso às ações do planejamento reprodutivo ${ }^{13}$.

Alinhada com os elementos constitutivos, atributos e estratégias das RAS no SUS ${ }^{15}$ a RC definiu ações para qualificação da gestão do cuidado por componentes e etapas de operacionalização. No âmbito da Governança da Rede, favoreceu a instituição dos Grupos Condutores Estaduais da Rede Cegonha (GCE-RC) com representação dos três entes federados: Secretaria Estadual de Saúde (SES), responsável pela coordenação geral do GCE-RC, Conselho de Secretarias Municipais de Saúde (COSEMS) e apoio institucional do MS, com as atribuições de: (1) mobilizar os dirigentes políticos do SUS em cada fase operacional da RC; (2); apoiar a organização dos processos de trabalho voltados à implantação/implementação da RC; (3) identificar e apoiar a solução de possíveis pontos críticos em cada fase da RC; e (4) monitorar e avaliar o processo de implantação/ implementação da $\mathrm{RC}^{13}$. 


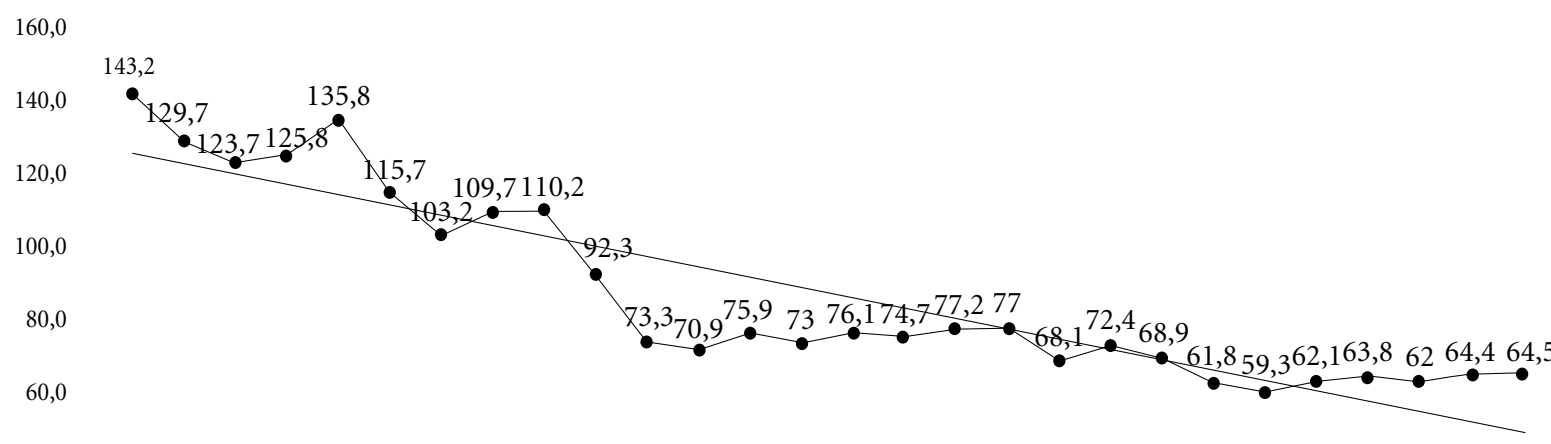

40,0

20,0

$0,0 \longdiv { 1 9 9 0 1 9 9 1 1 9 9 2 1 9 9 3 1 9 9 4 1 9 9 5 1 9 9 6 1 9 9 7 1 9 9 8 1 9 9 9 2 0 0 0 2 0 0 1 2 0 0 2 2 0 0 3 2 0 0 4 2 0 0 5 2 0 0 6 2 0 0 7 2 0 0 8 2 0 0 9 2 0 1 0 2 0 1 1 2 0 1 2 2 0 1 3 2 0 1 4 2 0 1 5 2 0 1 6 2 0 1 7 }$

Gráfico 1. Razão de Morte Materna no Brasil. 1990 a 2017. Ministério da Saúde, Brasil.

Fonte: DANTPS/SVS/MS ${ }^{14}$.

Considerando a operacionalização da RC em cinco fases (I - Adesão e diagnóstico; II - Desenho Regional da RC; III - Contratualização dos Pontos de Atenção; IV - Qualificação dos componentes e $\mathrm{V}$ - Certificação) ${ }^{13}$, o processo de monitoramento e avaliação das maternidades foi uma das responsabilidades dos GCE, visando verificar o cumprimento das ações de atenção à saúde em cada um dos componentes da Rede e, no caso deste estudo, o componente Parto e Nascimento.

De 2013 a 2015, o MS, em parceria com as Secretarias Estaduais (SES) e Municipais de Saúde (SMS), realizou o $1^{\circ}$ ciclo avaliativo das maternidades contempladas nos planos de ação regionais publicados até 2014, sendo as visitas avaliativas realizadas no período de 2014/15. Neste período, foram avaliadas três diretrizes em 250 serviços, sob condução dos GCE-RC e coordenação geral do MS. O $2^{\circ}$ ciclo teve início em 2015, com visitas avaliativas em 2016/17, sendo a apresentação e a discussão dos resultados avaliativos com os serviços em 2018/19. No $2^{\circ}$ ciclo foram avaliados 626 serviços; em 5 diretrizes, 17 dispositivos e 60 itens de verificação ${ }^{19}$. Diante da ampliação do escopo avaliativo, o MS estabeleceu parceria com instituições de pesquisa, Escola Nacional de Saúde Pública Sérgio Arouca (ENSP/Fiocruz) e a Universidade Federal do Maranhão (UFMA), ambas com experiências na avaliação do parto e nascimento.

A pergunta avaliativa que guiou o processo de monitoramento referiu-se ao grau de implantação das boas práticas na atenção ao parto e nascimento, e teve como um dos principais referenciais metodológicos o entendimento de que os processos avaliativos contribuem para a qualificação da gestão e da prática clínica. São muitas as finalidades da avaliação, "sejam elas oficiais ou oficiosas, explícitas ou implícitas, consensuais ou conflituosas, compartilhadas pela maioria dos atores ou por apenas alguns deles" ${ }^{20}$. No contexto da RC, destacaram-se as finalidades formativa e transformadora, ou seja, de fornecer informações estratégicas, capazes de apoiar tomadas de decisão para melhorar a intervenção no curso da atividade, utilizando o processo de avaliação como alavanca para transformar uma situação injusta ou problemática, pois a avaliação tem por objetivo a melhoria do bem-estar coletivo ${ }^{21}$.

A perspectiva da RC, ancorada na Política Nacional de Humanização (PNH), considera os processos avaliativos como espaços, momentos de debate e análise do próprio fazer em saúde. Um dos focos de análise (e de fomento) são as redes que se estabelecem como condição para as transformações no fazer em saúde. Como proposto na $\mathrm{RC}$, as três esferas de gestão (MS, SES e SMS) participaram do processo avaliativo, fortalecendo seu compromisso de governança da Rede.

Assim, a avaliação das maternidades foi realizada na relação direta com sua utilidade para a melhoria da prática cotidiana das políticas locais e da aprendizagem organizacional, com ganhos para os resultados pretendidos. Favorecendo o que Figueiró et al. ${ }^{22}$ destacam enquanto um dos 
aspectos dos modos de produção de conhecimento na utilização das avaliações, isto é, a avaliação com foco na utilidade, com o envolvimento dos interessados, gerando mudanças de pensamento, comportamento nas práticas e culturas institucionais, como resultado da aprendizagem durante o processo avaliativo.

As Oficinas de Devolutiva buscaram a indução da qualificação do modelo de atenção ao parto e nascimento e orientação da repactuação de compromissos firmados nos planos de ação regionais da RC. Trata-se de avaliação como instrumento de gestão do trabalho em saúde, possibilitando o desenvolvimento de competência coletiva, fundamental para a produção e reorganização de ações e serviços em saúde dirigidos às necessidades da população $0^{21,22}$.

Uma das questões centrais para o processo de implantação da RC foi a necessidade de organizá-la no contexto da regionalização da atenção à saúde e do princípio da cogestão ${ }^{23,24}$. Desde o início, o processo de implantação buscou estimular a criação de grupos condutores regionais e fomentar o desenvolvimento das etapas de análise diagnóstica, planejamento, implantação e avaliação da rede de forma partilhada entre o coletivo de trabalhadores dos diferentes pontos de atenção e gestores de cada região de saúde.

Nesse contexto, a proposta metodológica do processo de devolutiva da avaliação promoveu o envolvimento das diferentes categorias profissionais, pontos de atenção e gestores, contextualizando a discussão em cada região de saúde para a análise e possíveis pactuações para melhoria da qualidade da assistência.

Este artigo descreve e analisa o processo de devolutiva dos resultados do $2^{\circ}$ ciclo avaliativo das boas práticas na atenção ao parto e nascimento, em maternidades vinculadas à RC, a partir do alinhamento teórico metodológico de avaliação proposto, com enfoque no potencial de contribuição para a melhoria da gestão e atenção obstétrica e neonatal.

\section{Método}

Este artigo compõe o estudo avaliativo "Atenção ao parto e nascimento em maternidades vinculadas à Rede Cegonha", realizado no período de dezembro de 2016 a outubro 2017. A avaliação das maternidades foi realizada por Estimativa Rápida Participativa (ERP) ${ }^{25,26}$ para obter informações que reflitam as condições locais, a partir da ótica dos diferentes atores sociais envolvidos com o objeto avaliativo. Foram incluídos os hospitais públicos e mistos (privados conveniados ao SUS) que, em 2015, (i) realizaram 500 ou mais partos em região de saúde com plano de ação da RC, independentemente da liberação de recursos (n $=582$ ); e (ii) menos de 500 partos, em região de saúde com plano de ação da RC e com liberação de recursos $(n=24)$.

O processo avaliativo foi pactuado nos 27 Grupos Condutores Estaduais da RC, com participação de gestores locais nas diferentes etapas avaliativas: etapa 1 - Construção dos instrumentos de avaliação e organização do trabalho de campo; etapa 2 - Trabalho de campo e processamento dos dados; etapa 3 - Elaboração e validação da matriz avaliativa; etapa 4 - Classificação do grau de implantação; etapa 5 - Elaboração de relatórios estaduais e por maternidades.

Ações do processo avaliativo, enquanto estratégias de fortalecimento da governança tripartite da RC, que culminaram nas Oficinas de Devolutivas foram: (i) ofício do MS para as SES, COSEMS e SMS informando sobre os objetivos e resultados esperados do processo avaliativo, incluindo descrição de documentos necessários a serem analisados no momento da visita avaliativa; (ii) reunião com Grupos Condutores Estaduais da RC para alinhamento metodológico e compartilhamento de responsabilidades; (iii) definição de representantes das SES, COSEMS e SMS responsáveis pelo acompanhamento das visitas avaliativas por serviço; (iv) construção e acompanhamento tripartite de cronograma de visitas conjuntas (avaliadores e representantes do GCE-RC); (v) comunicação oficial aos serviços sobre o objetivo e data da visita avaliativa; (vi) visita avaliativa ao serviço; (vii) elaboração dos relatórios por serviço e Unidades Federadas (UF); (viii) reunião com o GCE-RC para apresentação e análise dos dados encontrados; (ix) reunião do GCE-RC para devolutiva e desdobramentos dos resultados com as maternidades; (x) Oficinas de Devolutivas com as maternidades.

$O$ processo de devolutiva com as maternidades compreendeu duas fases: a primeira, foi a apresentação dos resultados aos representantes do GCE-RC, secretarias de saúde e COSEMS, denominada reunião preparatória, com a finalidade de apresentar os resultados do estado e debater as principais características, potencialidades, desafios e desdobramentos sob responsabilidade dos gestores do SUS e, então, definir a estratégia para discussão com os serviços avaliados no estado. Nesta reunião foi acordada a participação das Instituições de Pesquisa, UFMA ou ENSP, em cada Oficina de Devolutiva e o envio de convites para os representantes dos GCE-RC, dos serviços - diretores e coordenações médicas e de enferma- 
gem das unidades de obstetrícia e neonatologia das maternidades. Os pontos importantes para a realização das Oficinas de Devolutiva foram: (i) apresentação dos resultados aos serviços coletivamente, agrupados por regiões de saúde, para fomentar a cooperação horizontal entre os serviços e mobilizar a articulação regional, incluindo a possibilidade de interação com os gestores nas Comissões Intergestores Regional (CIR) e Comissão Intergestores Bipartite (CIB); e (ii) a importância da agenda de continuidade do monitoramento e avaliação das Boas Práticas pelo GCE-RC, sendo apresentado pelo MS a proposta dos planos de ação das maternidades de acordo com os resultados alcançados no $2^{\circ}$ ciclo avaliativo.

A segunda fase do processo de devolutiva com as maternidades, foi denominada Oficinas de Devolutivas. Na abertura dessas Oficinas eram retomados os objetivos, princípios e diretrizes da $\mathrm{RC}$, o método e as etapas do processo avaliativo. Nas Oficinas foram apresentados dois tipos de relatórios: um de âmbito estadual e outro local, ou seja, para cada serviço avaliado. Os relatórios avaliativos elaborados para cada estado brasileiro contemplaram três aspectos: (i) características das maternidades, gestores, trabalhadores e puérperas participantes, (ii) resultados por diretriz, dispositivo e item de verificação por estado e maternidades. Cada maternidade recebeu os resultados por diretriz, dispositivo e item de verificação, de acordo com o formato de relatório do Quadro 1.

Nos relatórios por serviço foram apresentados os referenciais conceituais de cada diretriz com a respectiva classificação de acordo com o grau de implantação, seguida de campos abertos para a instituição registrar se aquele resultado estava adequado ao momento atual, as justificativas para a não adequação, as estratégias para melhoria, com a identificação dos responsáveis e prazos. Esse exercício coletivo, entre grupo da maternidade e representantes das secretarias de saúde, de comparar a situação encontrada no momento da avaliação com a situação atual, teve como objetivo a elaboração do plano de ação para qualificação da atenção ao parto e nascimento, e a continuidade de monitoramento pelos atores locais com apoio das áreas técnicas do MS.

Para a descrição e a análise do processo de devolutiva dos resultados, objeto deste artigo, foram utilizados dados quantitativos e qualitativos a partir de análise documental dos 27 relatórios estaduais das Oficinas de Devolutivas realizados pelas referências técnicas da Coordenação de Saúde das Mulheres e Saúde da Criança do MS. Nos relatórios constavam o desenho e a dinâmica de cada encontro, o número de participantes e represen- tação institucional, principais debates realizados, questionamentos, dúvidas e encaminhamentos.

A pesquisa foi aprovada pelo Comitê de Ética em Pesquisa com Seres Humanos da Universidade Federal do Maranhão e da Escola Nacional de Saúde Pública Sérgio Arouca, em 14 de dezembro de 2016.

\section{Resultados e discussão}

\section{Participação nas Oficinas de Devolutiva dos resultados}

As Oficinas de Devolutiva buscaram analisar os resultados com os gestores das secretarias de saúde e dos estabelecimentos de saúde a fim de propor agenda de trabalho intra e interinstitucionais com foco na melhoria da qualidade assistencial, por meio de estratégias de sustentabilidade de ações bem-sucedidas, e compartilhamento de responsabilidades frente à necessidade de ações para superação de dificuldades. Dificuldades estas, conforme destacadas em outros estudos, que demandam elaboração de estratégias de vigilância sistemática $^{27,28}$, análises coletivas ${ }^{29}$, construção de pertencimento e utilidade para os sujeitos envolvidos, capazes de representar a defesa de princípios éticos do direito à saúde universal e integral e à cidadania ${ }^{30}$ e, assim, superar os desafios da mortalidade materna e neonatal ${ }^{31}$.

De forma geral, as Oficinas transcorreram na seguinte sequência: (i) apresentação dos objetivos do processo avaliativo; (ii) método da avaliação; (iii) resultados do estado; (iv) discussão dos resultados por maternidades; (v) exercício analítico do relatório por serviço; e (vi) programação da agenda de trabalho para continuidade do monitoramento e avaliação das boas práticas. Alguns estados optaram, de acordo com o número de maternidades e distância entre os serviços, em fazer de forma concentrada na capital, mas, mesmo assim, foi mantida a análise por regiões de saúde. Alguns estados, representados pela SES e COSEMS, de forma propositiva, apresentaram um balanço das ações realizadas no âmbito da RC estadual e seus principais desafios.

Nas Oficinas de Devolutiva, participaram 1.641 profissionais entre secretários de saúde e técnicos das SES (405), SMS (287), COSEMS (33), Ministério Público Estadual (8), diretores e coordenadores médicos/enfermeiros das unidades de obstetrícia e neonatologia das maternidades (662), apoiadores institucionais dos Núcleos Estaduais do MS, representantes das Coordenações de Saúde das Mulheres e Saúde da Criança do MS 


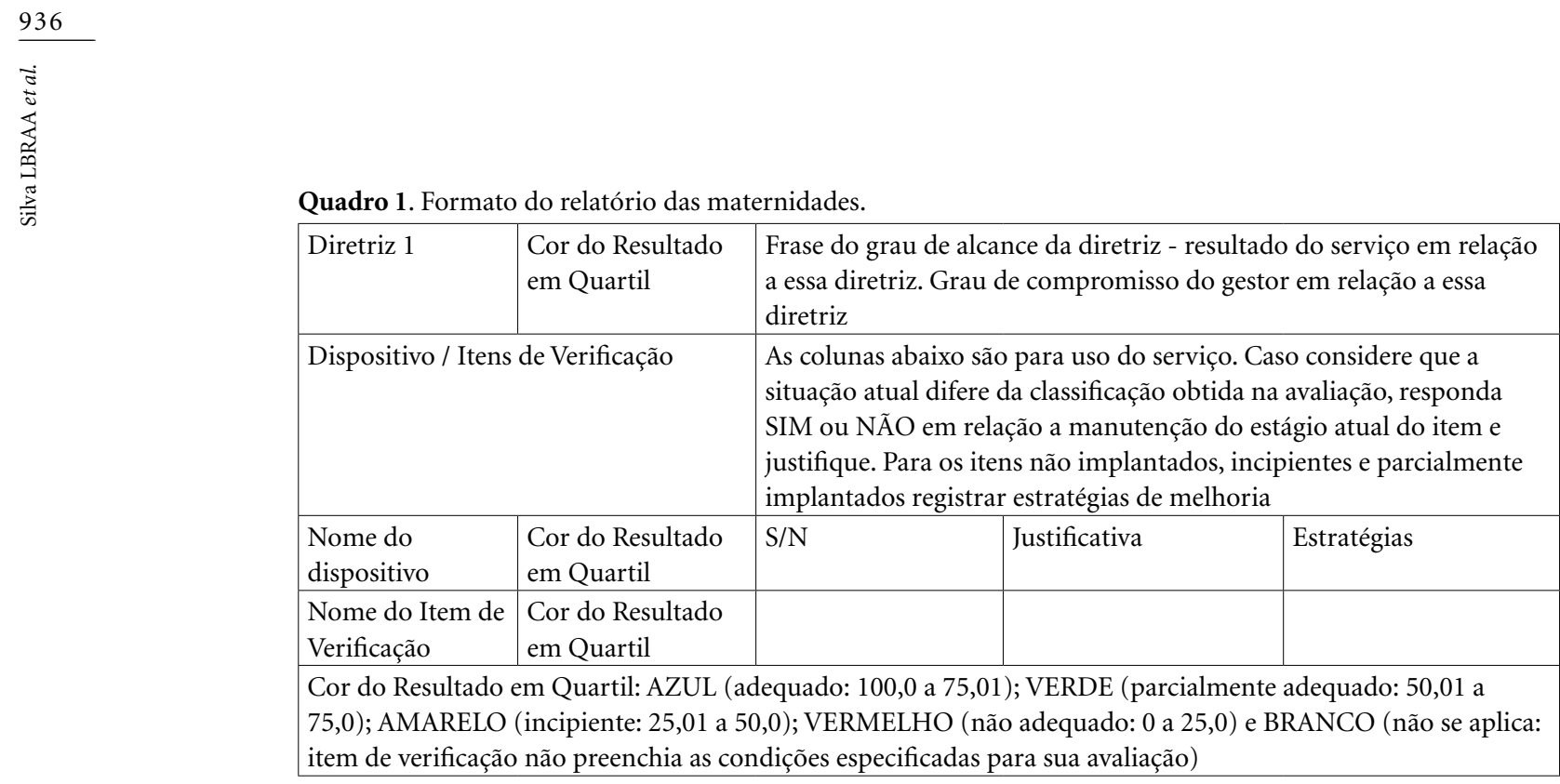

(107) e Universidade Federal do Maranhão ou ENSP/Fiocruz (28). De acordo com as listas de presença das Oficinas de Devolutivas foi possível identificar participação significativa dos gestores das maternidades ( $40 \%$ do total de participantes), entre diretores técnicos e coordenadores das áreas de obstetrícia e neonatologia/pediatria, com destaque para a presença dos coordenadores técnicos e profissionais enfermeiros, o que promoveu a qualificação do modelo assistencial ao parto e nascimento. Além disso, participaram representantes da Atenção Primária em Saúde (APS) e da gestão central das secretarias de saúde, o que permitiu o debate acerca dos desafios de integração entre os pontos de atenção para a integralidade do cuidado (Gráfico 2).

Merece destaque ainda, a participação do COSEMS em 17 UF. Tal participação, enquanto entidades que representam os entes municipais no âmbito estadual, contribui de forma sistêmica e potencializadora das ações e definições interfederativas no que cabe à política nacional e local de atenção ao parto e nascimento.

Vale ressaltar a participação em 8 UF de representantes do Ministério Público (MP) que favoreceu o intercâmbio com o SUS para uma atuação de forma solidária na proteção e defesa da saúde desenvolvendo atividades que contribuam para o efetivo respeito dos poderes públicos e dos serviços de relevância pública aos direitos à saúde e promover as medidas necessárias à sua garantia. Essa participação representa cooperação interinstitucional importante na busca pelo direito à cidadania e à saúde universal e integral, demonstrando aos gestores e profissionais do SUS e aos governantes o conceito operacional, am- plo e complexo do cuidado em saúde, como, por exemplo, a ação civil pública para a garantia das leis federais do direito ao acompanhante durante o parto ${ }^{32}$, direito da gestante ao conhecimento e a vinculação à maternidade onde receberá assistência no $\mathrm{SUS}^{33}$, as políticas públicas para a primeira infância ${ }^{34}$ e as leis estaduais (até 2019, 16 Estados promulgaram Leis Estaduais em relação ao parto e nascimento). A pluralidade e a heterogeneidade de representantes permitiu um debate reflexivo e direcionado às situações vivenciadas pelas instituições assistenciais e gestoras das três esferas de gestão. Esse efeito reflexivo e pragmático da diversidade de atores envolvidos com determinada política de saúde está em consonância com estudos que demonstram que a abrangência e a amplitude dos atores implicados no processo avaliativo favorece sua utilidade e capacidade de intervenção para a melhoria da assistência e o gerir o cuidado em saúde eq,30,35. $^{2}$.

\section{Temas principais sobre os modelos de atenção ao parto e nascimento}

Vale destacar os principais temas registrados nos relatórios estaduais que emergiram na discussão dos resultados da avaliação e seus desdobramentos. Questões relacionadas às maternidades de alto risco e suas especificidades, como a adoção das práticas recomendadas e a manutenção de intervenções desnecessárias, foram discutidas à luz das recomendações da $\mathrm{OMS}^{36}$ No debate junto aos serviços e gestores do SUS foi possível discutir barreiras para a implementação de modelo de atenção ao parto e nascimento preconizado pelas diretrizes nacionais e internacionais. $\mathrm{O}$ tema da 


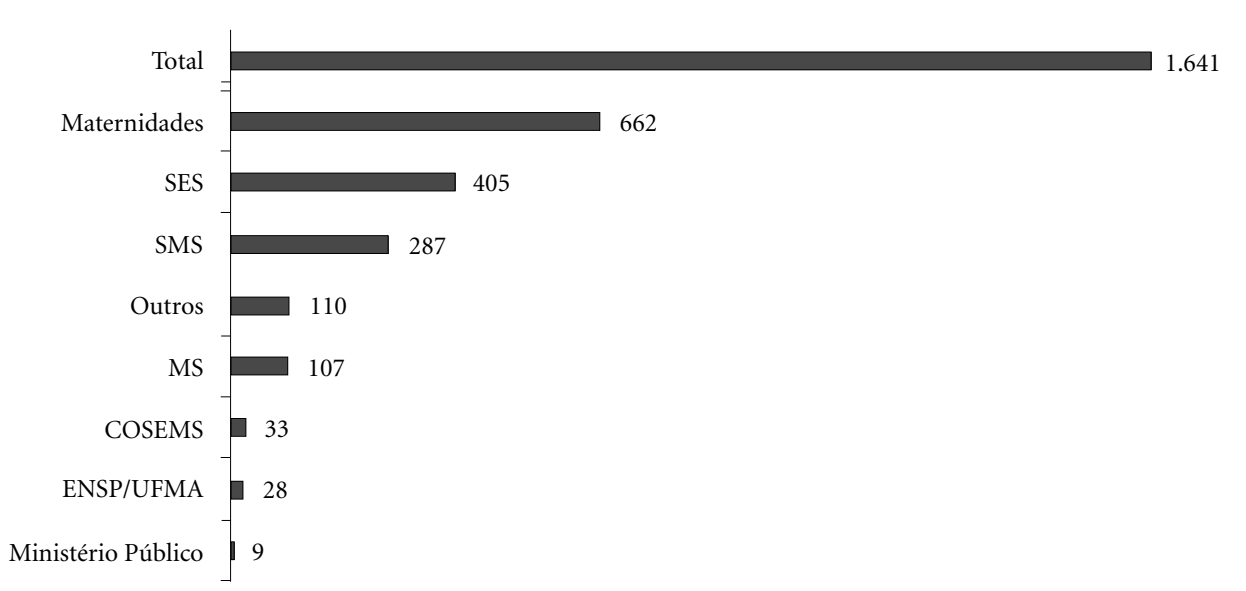

Gráfico 2. Representações institucionais nas Oficinas de Devolutivas. MS/Brasil, 2019.

Ministério Público: Representantes do Ministério Público do Estado

ENSP/UFMA: Representantes da Escola Nacional de Saúde Pública e Universidade Federal do Maranhão. COSEMS: representantes dos Conselhos de Secretários Municipais de Saúde nos Estados. MS: Representantes da Coordenação de Saúde da Criança e Coordenação de Saúde da Mulher. OUTROS: Outros participantes não especificados (Ex. profissionais que não preencheram o campo representação, representantes de conselhos e outras instituições não apuradas ou com participação incipiente). SMS: Representantes vinculados à Secretaria Municipal de Saúde. SES: Representantes vinculados à Secretaria Estadual de Saúde. Maternidades: Representantes das Maternidades Avaliadas.

insuficiência de profissionais e de recursos humanos no SUS perpassou as discussões sobre acolhimento e classificação de risco ${ }^{37}$ e ambiência, além da necessária reivindicação por maiores recursos materiais e financeiros para o SUS ${ }^{38}$.

Um dos efeitos ao apresentar e debater os resultados avaliativos sistematizados por diretrizes, dispositivos e itens de verificação em nível regional, foi elencar possibilidades de transformação no cotidiano das e entre as organizações e, consequentemente, em seus resultados, incluindo, não só os serviços assistenciais, mas também as instituições gestoras, como as Coordenações de Saúde das Mulheres e Saúde da Criança do MS, as Secretarias de Saúde e as instituições de pesquisa, como o Departamento de Epidemiologia da ENSP-Fiocruz e o Departamento de Saúde Pública da UFMA $^{29}$.

O agrupamento de maternidades por diretrizes, dispositivos e itens de verificação permitiu visualizar em uma matriz estadual o grau de implantação das boas práticas de atenção ao parto e nascimento com seus principais destaques, positivos ou negativos, e suas gradações, em duas perspectivas: (i) o conjunto das boas práticas implementadas por serviço; e (ii) o grau de implementação de cada diretriz e dispositivo no território (município, região de saúde, estado). Esta análise territorial favoreceu a reflexão das secretarias de saúde na implementação da política no seu território e na cooperação interinstitucional; por exemplo, análises sobre as facilidades e dificuldades no avanço para a implementação do acompanhante de escolha e em tempo integral e para o acolhimento e a classificação de risco em obstetrícia foram temas debatidos com intenso grau de corresponsabilização dos serviços e dos gestores das secretarias de saúde.

Os resultados avaliativos foram analisados tendo como ethos avaliativo que a informação produzida não deve ser utilizada como verdade absoluta, mas como ferramenta de negociação entre atores e interesses múltiplos, cuidando para assegurar análises contextualizadas e que dialoguem com as interpretações dos sujeitos envolvidos ${ }^{39}$.

As Oficinas permitiram o compartilhamento de dificuldades e facilidades entre gestores de serviços de características semelhantes sobre a implementação ou não de determinado dispositivo, como, por exemplo, o contato pele a pele e as implicações do modo de gerir e agir em saúde ${ }^{40}$.

Para tal efeito, foi proposta a modalidade de relatório que favorecesse a reflexão e o planejamento de estratégias para superação das principais dificuldades. Posteriormente à Oficina de Devolutiva, o relatório deveria ser elaborado com 
o coletivo de cada instituição a fim de elencar o plano de ação por maternidade para melhoria da qualidade assistencial e sustentabilidade das práticas exitosas. Esses planos de ação tornam-se instrumentos de monitoramento às maternidades e aos GCE-RC.

Até agosto de 2019 o MS recebeu 278 planos de ação de 11 estados, o que representa $1 / 3$ das Unidades Federadas e $46 \%$ das maternidades avaliadas. A elaboração desses planos estava norteada pelas diretrizes e dispositivos da $\mathrm{PNH}^{21}$ que visam à reorganização dos processos de trabalho em saúde, propondo centralmente transformações nas relações sociais entre trabalhadores e gestores em sua experiência cotidiana de organização e condução de serviços; transformações nas formas de produzir e prestar serviços à população; valorização da participação dos atores, o trabalho em equipe, com corresponsabilização de gestores, trabalhadores e usuários. Para propiciar essas mudanças, buscou-se refletir sobre o aumento da capacidade dos trabalhadores para analisar e intervir em seus processos de trabalho e na melhoria contínua da qualidade assistencial. Apostando na busca pela descentralização em saúde, na mediação entre a autonomia dos sujeitos e o controle das instituições (com suas normas e regras), refletindo sobre a potência de uma instituição também ser um espaço de possibilidades para a construção de estratégias que levem os trabalhadores a utilizar seu espaço privado de ação em favor do interesse público (dos usuários) ${ }^{29}$.

Apesar da participação significativa dos estados e dos atores envolvidos nas três esferas de gestão, mais da metade dos estados não conseguiu, até agosto de 2019, completar as etapas pactuadas da devolutiva, ou seja, o envio do plano de ação estadual ao MS. Trocas de gestão nos governos estaduais e dificuldades de agendas para pactuação desses planos nas CIR e CIB podem ser fatores que retardaram a finalização deste processo. Esse percentual de entrega dos planos de ação por Estado pode indicar a dificuldade por parte dos gestores das três esferas de gestão para a incorporação das práticas de monitoramento e avaliação como estratégias de qualificação da governança. Além de, também, poder indicar que o momento das Oficinas de Devolutiva não foi suficientemente mobilizador para reflexão e utilidade ${ }^{30}$ para aqueles estados que não conseguiram concluir a etapa de pactuação dos planos e envio ao MS.

Uma questão importante que não podemos deixar de analisar é o desafio da governança das redes de atenção à saúde nas instâncias decisórias do SUS que demanda um fortalecimento permanente das CIR, CIB e CIT, promovendo espaços de consenso que ocorrem por coordenação pluricêntrica, negociação, decisão por consenso, cooperação e interdependência e qualificação na elaboração e no uso dos instrumentos de gestão, dentre os planos de saúde, os planos regionais das redes, os contratos de gestão e, até mesmo, os planos de ação das maternidades ${ }^{41,42}$.

\section{Considerações finais}

Considerando que $68 \%$ das mortes maternas brasileiras são ainda associadas a causas obstétricas diretas e, portanto, evitáveis, devido principalmente às complicações hipertensivas e hemorrágicas, pode-se constatar que a qualidade da atenção está no cerne do problema da mortalidade materna no Brasil e indicam para os desafios da relação entre os componentes básicos da organização do sistema de saúde, as estratégias de qualificação e atualização científica dos profissionais de saúde. Promover espaços de diálogo plurais, participativos e prospectivos com a finalidade de melhoria da qualidade na atenção à saúde sob a análise das condições do sistema de saúde, organizado regionalmente, remete aos princípios e diretrizes constitutivos do SUS. Esta premissa permeia o desenho metodológico do processo avaliativo alinhado com as recomendações científicas de boas práticas de atenção ao parto e nascimento.

A incorporação de novos cuidados em saúde baseados em evidências científicas e garantia de direitos na atenção obstétrica e neonatal demandam sistemática periódica de visitas avaliativas, momentos de análise e reflexão coletiva dos resultados encontrados e o monitoramento e a avaliação dos planos de ação dos serviços. Assim, espera-se que novos ciclos sejam implementados e processos de monitoramento e avaliação da atenção obstétrica e neonatal incorporados ao cotidiano dos serviços e instituições de saúde. Espera-se, ainda, ampliar o escopo avaliativo para as situações de urgência e emergências obstétricas e as principais causas de morbimortalidade materna e neonatal. Também se faz necessária a ampliação das representações do controle social, por exemplo, conselhos de saúde e movimento de mulheres, como fontes de informação do processo avaliativo e sujeitos da transformação, por meio da participação nas Oficinas de Devolutivas dos resultados da atenção ao parto e nascimento.

Cabe destacar que o compartilhamento de representantes da APS com os serviços especializados favoreceu espaços de análise coletiva com potencial para definição de estratégias singulares e mais efetivas. Assim, espera-se a ampliação dessa 
experiência, com estímulo à participação, também, de representantes dos serviços especializados (maternidades, CPN) nos momentos de avaliação da APS no âmbito da atenção obstétrica e neonatal. Doravante, se reconhecerá que sistemas internacionais de saúde demonstram ser estratégicos para o alcance de resultados positivos à implementação de sistema informatizado da assistência clínica na gestação, parto, nascimento e puerpério, favorecendo a comunicação institucional e clínica mais horizontal e em tempo oportuno de acordo com as necessidades em saúde.

Por fim, ressalta-se a importância das instâncias de gestão do SUS em valorizar o interesse dos profissionais e gestores da saúde para a implementação de estratégias mais sustentáveis, visando à incorporação e apropriação da prática de monitoramento e avaliação nos serviços e entre eles.

\section{Colaboradores}

LBRAA Silva, A Angulo-Tuesta, MTR Massari, LCR Augusto, LLM Gonçalves, CKRT Silva e NP Minoia participaram da concepção, planejamento e análise dos dados; da redação ou revisão crítica da versão final e da aprovação final da versão a ser publicada; sendo responsáveis por todos os aspectos do trabalho na garantia da exatidão e integridade de seu conteúdo.

\section{Referências}

1. World Health Organization (WHO). Trends in maternal mortality: 1990 to 2015: estimates by WHO, UNICEF, UNFPA, World Bank Group and the United Nations Population Division. Geneve: WHO; 2015.

2. Leal MC, Pereira APE, Domingues RMSM, Theme Filha MM, Dias MAB, Nakamura-Pereira M, Bastos MH, Gama SGN. Intervenções obstétricas durante o trabalho de parto e parto em mulheres brasileiras de risco habitual. Cad Saude Publica 2014; 30(Supl. 1):s17-s32.

3. Carvalho EMP, Göttems LBD, Pires MRGM. Adesão às boas práticas na atenção ao parto normal: construção e validação de instrumento. Rev Esc Enferm USP 2015; 49(6):889-897.

4. Pacagnella RC, Nakamura-Pereira M, Gomes-Sponholz F, Aguiar RALP, Guerra GVDQL, Diniz CSG, Moraes Filho OB. Mortalidade materna no Brasil: propostas e estratégias para sua redução. Rev Bras Ginecol Obstet 2018; 40(09):501-506.

5. Szwarcwald CL, Escalante JJ, Rabello Neto DL, Souza Junior PR, Victora CG. Estimation of maternal mortality rates in Brazil, 2008-2011. Cad Saude Publica 2014; 30(Supl.1):s1-12.

6. Dias MAB, Domingues RMSM, Schilithz AOC, Nakamura-Pereira M, Diniz CSG, Brum IR, Martins AL, Theme Filha MM, Gama SGN, Leal MC. Incidência do near miss materno no parto e pós-parto hospitalar: dados da pesquisa Nascer no Brasil. Cad Saude Publica 2014; 30(Supl.1):s169-s181.
7. Lansky S, Friche AAL, Silva AAM, Campos D, Bittencourt SDA, Carvalho ML, Frias PG, Cavalcante RS, Cunha AJLA. Pesquisa Nascer no Brasil: perfil da mortalidade neonatal e avaliação da assistência à gestante e ao recém-nascido. Cad Saude Publica 2014; 30(Supl. 1):s192-s207.

8. Ventura M. A mortalidade materna: a persistente violação do direito de proteção da vida e autonomia feminina. Revista Bioética 2008; 16(2):217-228.

9. Leal MDC, Szwarcwald CL, Almeida PVB, Aquino EML, Barreto ML, Barros F, Victora C. Saúde reprodutiva, materna, neonatal e infantil nos 30 anos do Sistema Único de Saúde (SUS). Cien Saude Colet 2018; 23(6):1915-1928.

10. Brasil. Ministério da Saúde (MS). Departamento de Informação e Análise Epidemiológica. [Internet]. 2019 [acessado 2019 Jun 19]. Disponível em: http://svs. aids.gov.br/dantps/acesso-a-informacao/acoes-e-programas/buscaativa/indicadores-de-saude/mortalidade/

11. Organização das Nações Unidas Brasil. Transformando Nosso Mundo: A Agenda 2030 para o Desenvolvimento Sustentável [Internet]. 2015; [acessado 2019 Set 11]. Disponível em: https://nacoesunidas.org/ pos2015/agenda2030/

12. Souza JP. Mortalidade materna no Brasil: a necessidade de fortalecer os sistemas de saúde. Rev Bras Ginecol Obstet 2011; 33(10):273-279.

13. Brasil. Ministério da Saúde (MS). Portaria de Consolidação no 3 , de 28 de setembro de 2017. Consolidação das normas sobre as redes do Sistema Único de Saúde. Diário Oficial da União 2017; 3 out. 
14. Brasil. Ministério da Saúde (MS). Portaria de Consolidação $n^{\circ} 6$ de 28 de setembro de 2017. Consolidação das normas sobre o financiamento e a transferência dos recursos federais para as ações e os serviços de saúde do Sistema Único de Saúde. Diário Oficial da União, Brasília, DF, 03 de out. 2017. Suplemento, p.612. Título VIII, CAPÍTULO I, Seção I.

15. Brasil. Ministério da Saúde. Gabinete do Ministro. Portaria de Consolidação no 3 de 28 de setembro de 2017. Consolidação das normas sobre as redes do Sistema Único de Saúde. Diário Oficial da União, Brasília, DF, 03 de out. 2017. Suplemento, p. 193. Anexo I.

16. Brasil. Ministério da Saúde (MS). Gabinete do Ministro. Portaria de Consolidação no 3 de 28 de setembro de 2017. Consolidação das normas sobre as redes do Sistema Único de Saúde. Diário Oficial da União 2017; 3 out.

17. Organização Mundial da Saúde (OMS). Maternidade segura. Assistência ao parto normal: um guia prático. Genebra: OMS; 1996.

18. Brasil. Ministério da Saúde (MS). Diretrizes de Atenção à Gestante: a operação cesariana. Brasília: MS; 2016.

19. Brasil. Ministério da Saúde (MS). Diretrizes nacionais de assistência ao parto normal: versão resumida. Brasília: MS; 2017.

20. Bittencourt DAS, Vilela MEA. Atenção ao Parto e Nascimento em Maternidades da Rede Cegonha: avaliação do grau de implantação das ações. Cien Saude Colet 2021; 26(3):801-822.

21. Champagne F, Contandriopoulos AP, Brouselle A, Hartz Z, Denis JL. A Avaliação no Campo da Saúde. In: Brousselle A, Champagne F, Contandriopoulos AP, Hartz Z, organizadores. Avaliação: Conceitos e métodos. Rio de Janeiro: Fiocruz; 2011. p. 41-60.

22. Santos-Filho SB. Avaliação e Humanização em Saúde: aproximações metodológicas. $2^{\mathrm{a}}$ ed. Ijuí: UNIJUI; 2010.

23. Figueiró AC, Hart Z, Samico I, Cesse EAP. Usos e influência da avaliação em saúde em dois estudos sobre o Programa Nacional de Controle da Dengue. Cad Saude Publica 2012; 28(11):2095-2105.

24. Brasil. Ministério da Saúde (MS). Política Nacional de Humanização. HumanizaSUS: gestão participativa: cogestão. Brasília: MS; 2007.

25. Tanaka OY, Melo CMM. Uma proposta de abordagem transdisciplinar para avaliação em Saúde. Interface Botucatu 2000; 4(7):113-118.

26. Di Villarosa FNA. Estimativa Rápida e a divisão do território no Distrito Sanitário: Manual de Instruções. Brasília: OPS/OMS; 1993. (Série Desenvolvimento de Serviços de Saúde, n 11 ).

27. Piovesan MF, Padrão MVV, Dumont UM, Gondim GM, Flores O, Pedrosa JI, Lima LFM. Vigilância Sanitária: uma proposta de análise dos contextos locais. Rev Bras Epidemiol 2005; 8(1):83-95.

28. Geller SE, Koch AR, Garland CE, MacDonald EJ, Storey F, Lawton B. A global view of severe maternal morbidity: moving beyond maternal mortality. Reprod Health 2018; 15(Supl. 1):98.

29. Macfarlane A. Gissler M, Bolumar F, Rasmussen S. The availability of perinatal health indicators in Europe. Eur J Obstetr Gynecol Reproduc Biol 2003; 111(Supl. 1):S15-S32.
30. Merhy EE, Feuerwerker LCM. Novo olhar sobre as tecnologias de saúde: uma necessidade contemporânea. Merhy EE, Baduy RS, Seixas CT, Almeida DES, Slomp Junior H, organizadores. Avaliação compartilhada do cuidado em saúde: surpreendendo o instituído nas redes. Rio de Janeiro: Hexis; 2016. p. 59-72.

31. Nemes MIB. Avaliação de serviços de saúde: a experiência do Qualiaids. Porto Alegre: Rede Unida; 2016.

32. Pasche DF, Vilela MEA, Di Giovanni M, Almeida PVB, Franco Netto TL. Rede Cegonha: desafios de mudanças culturais nas práticas obstétricas e neonatais. Divulg Saude Debate 2014; 52:58-71.

33. Brasil. Lei $\mathrm{n}^{\circ} 11.108$, de 7 de abril de 2005. Altera a Lei no 8.080 , de 19 de setembro de 1990, para garantir às parturientes o direito à presença de acompanhante durante o trabalho de parto, parto e pós-parto imediato, no âmbito do Sistema Único de Saúde - SUS. Diário Oficial da União 2005; 7 abr.

34. Brasil. Lei $\mathrm{n}^{\circ} 11.634$, de 27 de dezembro de 2007. Dispõe sobre o direito da gestante ao conhecimento e a vinculação à maternidade onde receberá assistência no âmbito do Sistema Único de Saúde. Diário Oficial da União 2007; 27 dez.

35. Brasil. Lei $\mathrm{n}^{\circ} 13.257$, de 5 de março de 2016. Dispõe sobre as políticas públicas para a primeira infância. Diário Oficial da União 2016; 5 mar.

36. Furtado JP, Onocko-Campos RT, Moreira MIB, Trape TL. A elaboração participativa de indicadores para a avaliação em saúde mental. Cad Saude Publica 2013; 29(1):102-110.

37. World Health Organization (WHO). Recommendations: intrapartum care for a positive childbirth experience. Geneva: WHO; 2018.

38. Figueiroa MN, Menezes MLB, Monteiro EMLM, Aquino JM, Mendes NOG, Silva PVT. Acolhimento do usuário e classificação de risco em emergência obstétrica: avaliação da operacionalização em maternidade -escola. Rev Esc Anna Nery 2017; 21:4.

39. Carvalho ALB, Souza MF, Shimizu HE, Senra IMVB, Oliveira KC. A gestão do SUS e as práticas de monitoramento e avaliação: possibilidades e desafios para a construção de uma agenda estratégica. Cien Saude Colet 2012; 17(4):901-911.

40. Deslandes SF, Assis SG. Abordagens quantitativa e qualitativa em saúde: o diálogo das diferenças. In: Minayo MCS, Deslandes SF, organizadores. Caminhos do pensamento: epistemologia e método. Rio de Janeiro: Fiocruz; 2002. p. 195-226.

41. Brasil. Ministério da Saúde (MS). Além da sobrevivência: práticas integradas de atenção ao parto, benéficas para a nutrição e a saúde de mães e crianças. Brasília: MS; 2013.

42. Mendes EV. Comentários sobre as Redes de Atenção à Saúde no SUS. Saúde Debate 2014; 52:38-49.

43. Carvalho ALB; Jesus WLA, Senra IMVB. Regionalização no SUS: processo de implementação, desafios e perspectivas na visão crítica de gestores do sistema. Cien Saude Colet 2017; 22(4):1155-1164.

Artigo apresentado em 13/04/2020

Aprovado em 05/08/2020

Versão final apresentado em 07/08/2020

Editores-chefes: Romeu Gomes, Antônio Augusto Moura da Silva 